\section{Interne Desynchronisation}

Helga Peter ${ }^{1}$ und Thomas Penzel ${ }^{2}$

${ }^{1}$ Marburg, Deutschland

${ }^{2}$ Interdisziplinäres Schlafmedizinisches Zentrum, Charité Universitätsmedizin Berlin, Berlin, Deutschland

\section{Synonyme}

Desynchronisation endogener Rhythmen; interne Dissoziation

\section{Englischer Begriff}

internal desynchronisation

\section{Definition}

Sie kann bezüglich der zirkadianen Rhythmik ausgelöst werden, wenn Versuchspersonen über längere Zeiträume unter experimentellen Bedingungen der zeitgeberfreien Isolation leben, beispielsweise in sog. Bunkerversuchen. Sie tritt im Rahmen von Zirkadianen Rhythmusschlafstörungen auf, beispielsweise bei „Jetlag“ oder Schichtarbeit. Siehe , „Nachtarbeit und Schichtarbeit“". 